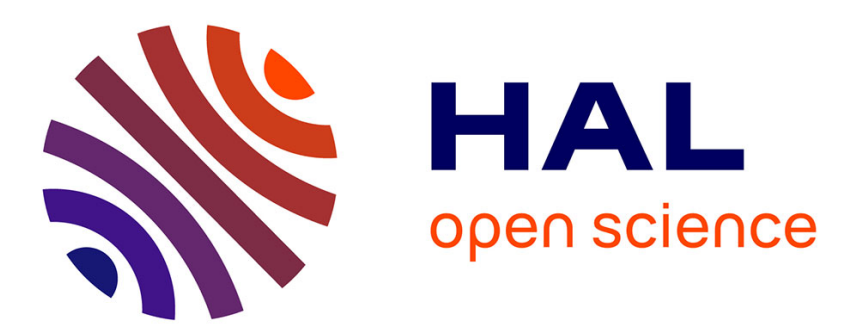

\title{
Études théorique et expérimentale de plusieurs régimes permanents de diffusion déterminés par la précipitation mutuelle de deux réactifs
}

Jean Salvinien, Jean Jacques Moreau, R. Gaufrès

\section{To cite this version:}

Jean Salvinien, Jean Jacques Moreau, R. Gaufrès. Études théorique et expérimentale de plusieurs régimes permanents de diffusion déterminés par la précipitation mutuelle de deux réactifs. Journal de Chimie Physique et de Physico-Chimie Biologique, 1953, 50, pp.1 - 5. 10.1051/jcp/1953500001 . hal-01738685

\section{HAL Id: hal-01738685 \\ https://hal.science/hal-01738685}

Submitted on 20 Mar 2018

HAL is a multi-disciplinary open access archive for the deposit and dissemination of scientific research documents, whether they are published or not. The documents may come from teaching and research institutions in France or abroad, or from public or private research centers.
L'archive ouverte pluridisciplinaire HAL, est destinée au dépôt et à la diffusion de documents scientifiques de niveau recherche, publiés ou non, émanant des établissements d'enseignement et de recherche français ou étrangers, des laboratoires publics ou privés. 


\title{
ETUDES THEORIQUE ET EXPERIMENTALE DE PLUSIEURS RÉGIMES PERMANENTS DE DIFFUSION DETERMINES PAR LA PRÉCIPITATION MUTUELLE DE DEUX RÉACTIFS
}

\author{
par J. SALVINIEN, J. J. MOREAU et R. GAUFRÈS
}

Manuscrit reçu le 1-10-52

\section{SOMMAIRE}

Nous plaçant dans des conditions idéales simples, nous donnons la théorie mathématique de plusieurs régimes permanents de diffusion déterminés par la précipitation mutuelle de deux réactifs. A condition de choisir des systèmes dépourvus de complications, on obtient une bonne vérification expérimentale des résultats obtenus. Ces derniers peuvent servir de base à des mesures relatives de coefficients de diffusion ou à l'étude de phénomènes plus compliqués.

\section{Introduction.}

L'histoire expérimentale de la diffusion peut se diviser en deux périodes.

Dans une première période, on a supposé que le coefficient de diffusion, défini par la relation :

$$
d \mathrm{Q}=-\mathrm{DS} \frac{\partial \mathrm{C}}{\partial x} d t
$$

est indépendant de la concentration $\mathrm{C}$ et du gradient de concentration $\frac{\partial \mathrm{C}}{\partial x}$. On a ainsi créé un domaine idéal, assez proche du domaine réel, et facilement accessible aux mathématiciens. De la sorte, il a été possible de poser et de résoudre un certain nombre de problèmes classiques par application de la loi de FICK :

$$
\frac{\partial \mathrm{C}}{\partial t}=\mathrm{D}\left(\frac{\partial^{2} \mathrm{C}}{\partial x^{2}}+\frac{\partial^{2} \mathrm{C}}{\partial y^{2}}+\frac{\partial^{2} \mathrm{C}}{\partial z^{2}}\right)
$$

qui se démontre aisément à partir de la relation (1). Les solutions les plus commodes de ces problèmes ont permis la mesure d'un grand nombre de coefficients de diffusion.

Dans la période actuelle, les mesures sont devenues beaucoup plus précises. On s'est aperçu que les coefficients de diffusion varient légèrement avec la concentration et peut-être avec son gradient. L'étude de ces variations est en plein essor à l'heure actuelle. Quand la diffusion s'effectue dans un gel, les phénomènes se compliquent car il peut y avoir adsorption préférentielle d'une molécule ou d'un ion, désolvata- tion partielle des ions en présence du colloïde hydrophile, protection du corps insoluble formé par double décomposition, etc... Bref, comme il arrive obligatoirement dans les sciences physiques, le domaine réel est beaucoup plus vaste et plus compliqué que le domaine idéal primitivement créé

Cependant, ce domaine idéal mérite encore d'être exploré, ne serait-ce que pour permettre la mise en évidence de nouveaux écarts entre l'expérience et une théorie un peu trop rudimentaire.

Nous indiquons, dans cet article, la solution d'un groupe de problèmes relatifs à des diffusions dont le régime permanent est déterminé par la précipitation mutuelle de deux réactifs. A condition de faire un choix judicieux du gel et des corps diffusants, les résultats donnés par la vieille théorie classique sont convenablement vérifiés par l'expérience.

\section{Problème général en régime permanent.}

Deux corps purs $R_{1}$ et $R_{2}$, réagissant l'un sur l'autre pour former un précipité quasi insoluble et non protégé, sont mis à diffuser dans un massif de gel homogène. Ils partent respectivement de deux portions $S_{1}$ et $S_{2}$ de la frontière du massif. Sur $S_{1}$ et $S_{2}$, les concentrations $C_{1}$ et $C_{2}$ en réactifs $R_{1}$ et $R_{2}$ sont maintenues constantes. Eventuellement, le



Fig. 1. reste $S_{3}$ de la frontière est isolé. Au bout d'un certain temps, la diffusion est assez avancée pour qu'en différents points du gel, le produit. de solubilité soit atteint. Le précipité apparaît sous forme d'un front $F$ (fig. 1). Par suite de la dissymétrie des apports en $R_{1}$ et $R_{2}, F$ se déplace en général. Mais si les concentrations $C_{1}$ et $C_{2}$ ne sont pas trop différentes l'une de l'autre, on constate que $\mathrm{F}$ finit par se stabiliser entre $S_{1}$ et $S_{2}$. Un régime permanent s'établit. 
Le front $F$ partage le massif en deux domaines $\delta_{1}$ et $\delta_{2}$. Le domaine $\delta_{1}$, contigu à $\mathrm{S}_{1}$, contient uniquement du réactif $R_{1}$ dont la concentration $\gamma_{1}$ varie d'un point à l'autre. De même, le domaine $\delta_{2}$, contigu à $S_{2}$, renferme le seul réactif $R_{2}$ avec la concentration variable $\gamma_{2}$.

Dans ces domaines respectifs, $\gamma_{1}$ et $\gamma_{2}$ vérifient l'équation de Fick :

$$
\begin{aligned}
& \frac{\partial \gamma_{1}}{\partial t}=D_{1} \Delta \gamma_{1} \\
& \frac{\partial \gamma_{2}}{\partial t}=D_{2} \Delta \gamma_{2}
\end{aligned}
$$

$D_{1}$ et $D_{2}$ désignent les coefficients de diffusion de $R_{1}$ et de $R_{2}$.

Quand le régime permanent s'est établi, les dérivées de $\gamma_{1}$ et $\gamma_{2}$ par rapport au temps sont nulles. Les équations (3) et (4) se réduisent à des équations de LAPLAGE :

$$
\Delta \gamma_{1}=0 \text { dans } \delta_{1}
$$

et :

$$
\Delta \gamma_{2}=0 \text { dans } \delta_{2}
$$

A ces conditions indéfinies vérifiées dans la masse, nous devons ajouter les conditions aux limites suivantes :

$$
\begin{aligned}
& \gamma_{1}=C_{1} \text { sur } S_{1} \\
& \gamma_{2}=C_{2} \text { sur } S_{2}
\end{aligned}
$$

Sur la surface isolée $S_{3}$, éventuellement, nous devons exprimer la nullité des flux, ce qui revient, d'après la relation (1), à écrire que les dérivées normales des concentrations sont nulles :

$$
\begin{aligned}
& \frac{d \gamma_{1}}{d n}=0 \text { sur } \mathrm{S}_{3} \\
& \frac{d \gamma_{2}}{d n}=0 \text { sur } \mathrm{S}_{3}
\end{aligned}
$$

Enfin, sur le front $F$, dont la position est inconnue a priori, nous écrivons :

$\left.1^{\circ}\right)$ que les concentrations des réactifs sont nulles :

$$
\begin{aligned}
& \gamma_{1}=0 \operatorname{sur} F \\
& \gamma_{2}=0 \operatorname{sur} \cdot F
\end{aligned}
$$

$2^{\circ}$ ) que les flux des réactifs sont égaux et opposés puisque le front est immobile. Naturellement, comme ces flux se neutralisent par réaction chimique, il est nécessaire d'exprimer les concentrations en valencesgramme par unité de volume. La relation (1) donne alors :

$$
\mathrm{D}_{1} \frac{d \gamma_{1}}{d n}=-\mathrm{D}_{2} \frac{d \gamma_{2}}{d n} \operatorname{sur} \mathrm{F}
$$

La détermination des fonctions $\gamma_{1}$ et $\gamma_{2}$ et du front permanent de précipitation $F$ peut être rattachée à un problème préliminaire plus simple que voici :

Déterminer une fonction $U$ par les conditions suivantes :

$$
\Delta \mathrm{U}=0
$$

dans tout le domaine.

$$
\begin{gathered}
\mathrm{U}=\mathrm{U}_{1} \text { sur } \mathrm{S}_{1} \\
\mathrm{U}=\mathrm{U}_{2} \text { sur } \mathrm{S}_{2} \\
\frac{d \mathrm{U}}{d n}=0 \operatorname{sur} \mathrm{S}_{3}
\end{gathered}
$$

$\mathrm{U}_{1}$ et $\mathrm{U}_{2}$ étant des constantes choisies, suivant les cas, pour la commodité du calcul.

Ce problème mixte harmonique peut être résolu mathématiquement dans de nombreux cas simples. En toute généralité, on pourrait également évaluer la fonction U par analogie rhéólectrique, puisque U n'est autre que le potentiel électrique aux divers points d'un conducteur géométriquement semblable au massif étudié, maintenu à des potentiels constants par $\mathrm{S}_{1}$ et $\mathrm{S}_{2}$ et isolé par $\mathrm{S}_{3}$.

$\mathrm{U}$ étant supposée connue, cherchons à satisfaire les conditions (5) à (12) en prenant :

$$
\begin{aligned}
& \gamma_{1}=A_{1} U+B_{1} \\
& \gamma_{2}=A_{2} U+B_{2}
\end{aligned}
$$

$A_{1}, B_{1}, A_{2}, B_{2}$ étant des constantes à déterminer. Nous essayons alors de voir si $F$ ne coïncide pas avec l'une des surfaces équipotentielles :

$$
\mathrm{U}=\mathrm{U}_{\mathrm{o}}
$$

$\mathrm{U}_{\mathrm{o}}$ désignant une constante également inconnue.

Grâce aux conditions (14) et (17), les conditions (5) (6) et (9) (10) sont évidemment remplies.

D'après (15) et (16), les conditions (7) (8) appliquées aux expressions (18) et (19) de $\gamma_{1}$ et $\gamma_{2}$ donnent:

$$
\begin{aligned}
& \mathrm{C}_{1}=\mathrm{A}_{1} \mathrm{U}_{1}+\mathrm{B}_{1} \\
& \mathrm{C}_{2}=\mathrm{A}_{2} \mathrm{U}_{2}+\mathrm{B}_{2}
\end{aligned}
$$

(11) et (12) imposent :

$$
\begin{aligned}
& A_{1} U_{0}+B_{1}=0 \\
& \Lambda_{2} U_{0}+B_{2}=0
\end{aligned}
$$

Enfin, la condition (13) d'égalité des flux entraîne :

soit :

$$
\mathrm{D}_{1} \mathrm{~A}_{1} \frac{d \mathrm{U}}{d n}=-\mathrm{D}_{2} \mathrm{~A}_{2} \frac{d \mathrm{U}}{d n}
$$

$$
D_{1} A_{1}=-D_{2} A_{2}
$$

Les cinq dernières relations déterminent exactement les cinq constantes $A_{1}, B_{1}, A_{2}, B_{2}$ et $U_{0}$. Le 
problème se trouve résolu. L'élimination de $\mathrm{A}_{1}, \mathrm{~B}_{1}$, $\mathrm{A}_{2}$ et $\mathrm{B}_{2}$ donne :

$$
\frac{\mathrm{D}_{1}}{\mathrm{D}_{2}}=-\frac{\mathrm{C}_{2}}{\mathrm{C}_{1}} \frac{\mathrm{U}_{1}-\mathrm{U}_{0}}{\mathrm{U}_{2}-\mathrm{U}_{0}}
$$

L'équation (26) relie la position du front de régime permanent, déterminée par la valeur de Uo, au rapport des coefficients de diffusion des deux réactifs. Par conséquent, elle doit permettre, dans les cas particuliers commodes, de faire des mesures relatives des coefficients de diffusion.

\section{Cas particuliers aisément calculables.}

Nous nous limitons à des gels coulés en couches d'épaisseur faible et constante (environ $2 \mathrm{~mm}$.) sur des plaques de verre planes. Le phénomène est sensiblement uniforme dans toute l'épaisseur de la couche et l'étude mathématique est celle d'un problème de diffusion dans un plan avec deux coordonnées $x, y$ seulement.

\section{$\left.1^{\circ}\right)$ Domaine rectangulaire $(*)$.}

Le côté $\mathrm{P}_{1} \mathrm{P}_{1}^{\prime}(x=$ a) correspond à $\mathrm{S}_{1}$



Fig. 2. (fig. 2). Le côté opposé $\mathrm{P}_{2} \mathrm{P}_{2}^{\prime}(x=+a)$ correspond à $\mathrm{S}_{2}$. On indiquera plus loin la réalisation expérimentale de concentrations uniformes données $\mathrm{C}_{1}$ et $\mathrm{C}_{2}$ des réactifs $R_{1}$ et $R_{2}$ sur ces deux segments. Les côtés $\mathrm{P}_{1} \mathrm{P}_{2}$ et $\mathrm{P}_{1}^{\prime} \mathrm{P}_{2}^{\prime}$ sont isolés et constituent la partie $\mathrm{S}_{3}$ de la frontière.

La fonction choisie :

$$
\mathrm{U}=x
$$

satisfait aux conditions (14), (15), (16) et (17). Elle prend la valeur $\mathrm{U}_{1}=-a$ sur $\mathrm{P}_{1} \mathrm{P}_{1}^{\prime}$ et la valeur $\mathrm{U}_{2}=+a \operatorname{sur} \mathrm{P}_{2} \mathrm{P}_{2}^{\prime}$. Le front permanent s'établit sur le segment $Q^{\prime}$ d'abscisse $x=\mathrm{U}_{0}$.

D'après (26), on a :

$$
\frac{\mathrm{D}_{1}}{\mathrm{D}_{2}}=\frac{\mathrm{C}_{2}}{\mathrm{C}_{1}} \frac{a+\mathrm{U}_{0}}{a-\mathrm{U}_{0}}=\frac{\mathrm{C}_{2}}{\mathrm{C}_{1}} \frac{\mathrm{QP}_{1}}{\mathrm{QP}_{2}}
$$

Les mesures de $\mathrm{QP}_{1}$ et $\mathrm{QP}_{2}$ permettent de calculer $\frac{\Gamma_{1}}{D_{2}}$.

(*) On retrouve le problème du mur, classique dans l'étude de la propagation de la chaleur.

$\left.2^{\circ}\right)$ Domaine angulaire, éventuellement limité par des arcs de cercle concentriques.

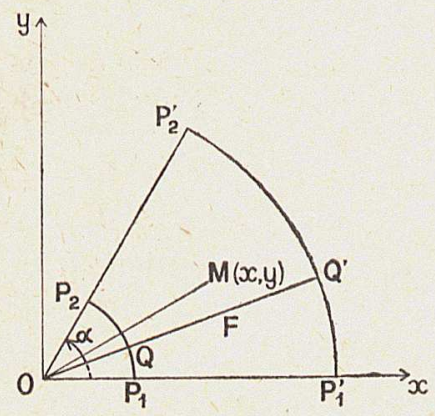

Fig. 3.
Les segments $P_{1}$ $\mathrm{P}_{1}^{\prime}$ et $\mathrm{P}_{2} \mathrm{P}_{2}^{\prime}$ correspondent respectivement à $S_{1}$ et $S_{2}$ et font entre eux l'angle $\alpha$ de sommet $\mathrm{O}$ (fig. 3 ). La frontière isolée $\mathrm{S}_{3}$ est formée des deux arcs de cercles $\mathrm{P}_{1} \mathrm{P}_{2}$ et $\mathrm{P}_{1}^{\prime} \mathrm{P}_{2}^{\prime}$ concentriques à $\mathrm{O}$. On choisit pour U l'angle :

$$
\mathrm{U}(x, y)=(\mathrm{O} x, \mathrm{OM})=\operatorname{arctg} \frac{y}{x}
$$

qui est bien une fonction harmonique et satisfait aux conditions (15), (16) et (17) avec $U_{1}=O$ sur $\mathrm{P}_{1} \mathrm{P}_{1}^{\prime}$ et $\mathrm{U}_{2}=\alpha$ sur $\mathrm{P}_{2} \mathrm{P}_{2}^{\prime}$.

Le front s'établit donc sur le segment radial $Q Q^{\prime}$ correspondant à la valeur $\mathrm{U}_{0}=(\mathrm{O} x, \mathrm{OQ})$. La relation (26) devient :

$$
\frac{D_{1}}{D_{2}}=\frac{\mathrm{C}_{2}}{\mathrm{C}_{1}} \frac{\mathrm{U}_{0}}{\alpha-\mathrm{U}_{0}}=\frac{\mathrm{C}_{2}}{\mathrm{C}_{1}} \frac{\left(\mathrm{OP}_{1}, \mathrm{OQ}\right)}{\left(\mathrm{OQ}, \mathrm{OP}_{2}\right)}
$$

\section{$\left.3^{\circ}\right)$ Domaine extérieur à deux cercles.}

Par un procédé qui sera précisé ultérieurement, les concentrations $\mathrm{C}_{1}$ et $\mathrm{C}_{2}$ sont maintenues constantes sur deux cercles $S_{1}$ et $S_{2}$ extérieurs l'un à l'autre (fig. 4).

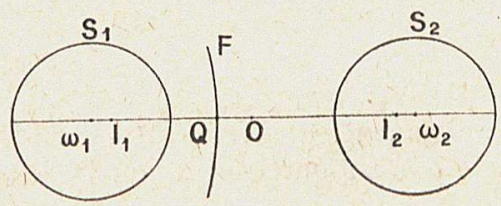

Fig. 4.

La fonction U se construit de la manière suivante :

Si l'on introduit deux points fixes $\mathrm{I}_{1}$ (coordonnées $\left.x_{1}, y_{1}\right)$ et $I_{2}$ (coordonnées $\left(x_{2}, y_{2}\right)$ et que l'on pose :

$$
\begin{aligned}
& \mathrm{MI}_{1}=\rho_{1} \\
& \mathrm{MI}_{2}=\rho_{2}
\end{aligned}
$$

les deux fonctions :

et :

$$
\log p_{1}=\log \sqrt{\left(x-x_{1}\right)^{2}+\left(y-y_{1}\right)^{2}}
$$

$$
\log p_{2}=\log \sqrt{\left(x-x_{2}\right)^{2}+\left(y-y_{2}\right)^{2}}
$$


sont harmoniques, de mème que leur différence :

$$
U=\log p_{1}-\log p_{2}=\log \frac{p_{1}}{\rho_{2}}
$$

Pour la commodité du calcul numérique, on pourra supposer qu'il s'agit ici de logarithmes décimalux.

Afin de satisfaire aux conditions (15) et (16), nous prendrons pour $\mathrm{I}_{1}$ et $\mathrm{I}_{2}$ les points limites du faisceau de cercles défini par $S_{1}$ et $S_{2}$, de sorte que le rapport des distances :

$$
\frac{\rho_{1}}{\rho_{2}}=\frac{M I_{1}}{M I_{2}}
$$

prend les valeurs constantes respectives $k_{1}$ et $k_{2}$ sur ces deux cercles. Donc :

$$
\begin{aligned}
& U_{1}=\log k_{1} \\
& U_{2}=\log k_{2}
\end{aligned}
$$

Le front permanent s'établit sur un autre cercle du faisceau défini par :

$$
\frac{\mathrm{MI}_{1}}{\mathrm{MI}_{2}}=k_{0}
$$

avec :

$$
\mathrm{U}_{0}=\log k_{0}
$$

D'après (26), il vient :

$$
\frac{\mathrm{D}_{1}}{\mathrm{D}_{2}}=-\frac{\mathrm{C}_{2}}{\mathrm{C}_{1}} \frac{\log k_{1}-\log k_{0}}{\log k_{2}-\log k_{0}}
$$

Achevons d'expliciter les éléments géométriques du problèmes dans le cas usuel où les deux cercles $\mathrm{S}_{1}$ et $\mathrm{S}_{2}$ ont le même rayon $r$ (fig. 5 ).

Soit :

$$
\omega_{1} \omega_{2}=2 l
$$

la distance des centres. Les points $I_{1}$ et $I_{2}$ sont alors symétriques par rapport au milieu $O$ de $\omega_{1} \omega_{2}$ avec :

$$
\mathrm{OI}_{1}=\mathrm{OI}_{2}=\sqrt{l^{2}-r^{2}}
$$

Cette mise en place est à effectuer une fois pour toutes de même que le calcul de $k_{1}$ :

$$
k_{1}=\frac{1}{k_{2}}=\frac{l-\sqrt{l^{2}-r^{2}}}{r}
$$

si l'appareillage est construit de telle sorte que $r$ et $l$ soient invariables.

Le front $F$ coupe $\omega_{1} \omega_{2}$ au point $Q$ et l'on obtient :

$$
k_{0}=\frac{\mathrm{QI}_{1}}{\mathrm{QI}_{2}}
$$

par une mesure facile.

\section{Vérifications expérimentales des solutions particu- lières.}

Jusqu'ici, nous nous sommes bornés à la vérification des formules données par la théorie. Afin d'obtenir des résultats comparables, nous avons choisi les mèmes réactifs pour les trois cas particuliers : à savoir $\mathrm{Cl}_{2} \mathrm{Ba}$ comme réactif $\mathrm{R}_{1}$ et $\mathrm{SO}_{4} \mathrm{~K}_{2}$ comme réactif $R_{2}$. Le précipité de sulfate de baryum n'est pas protégé par la gélatine. Celle-ci est purifiée de telle sorte que les ions gênants $\mathrm{Cl}^{-}$et $\mathrm{Ca}^{++}$ont pratiquement disparu.

L'expérience nous a montré, qu'avec les réactifs précédents, le front de régime permanent coïncide à peu près avec le front de première précipitation si les concentrations $\mathrm{C}_{1}$ et $\mathrm{C}_{2}$ sont égales.

\section{0) Domaine rectangulaire.}

Dans la lame gélatineuse de $2 \mathrm{~mm}$. d'épaisseur, coulée sur une plaque de verre $9 \times 12$, on ménage deux réservoirs rectangulaires étroits, de $8 \mathrm{~cm}$. de long, parallèles aux petits côtés de la plaque et situés à $15 \mathrm{~mm}$. environ l'un de l'autre. Les côtés en regard $S_{1}$ et $S_{2}$ des réservoirs doivent être bien nets et rectilignes. On les taille avec la lame d'un rasoir à dissection. Les réservoirs sont remplis respectivement avec les solutions des réactifs $R_{1}$ et $\mathrm{R}_{2}$. Les concentrations $\mathrm{C}_{1}$ et $\mathrm{C}_{2}$ sont maintenues constantes par agitation et renouvellement. Des précautions classiques évitent les variations de température et le dessèchement de la gélatine.

\section{Front de première précipitation.}

Au bout d'un temps de l'ordre de quelques heures, apparaît un front rectiligne de première précipitation, parallèle aux bords $S_{1}$ et $S_{2}$ des réservoirs. L'expérience montre que sa position, contrairement à celle $\mathrm{du}$ front de régime permanent, dépend peu du rapport $\mathrm{C}_{1} / \mathrm{C}_{2}$. Ce résultat, qui peut sembler assez paradoxal a priori, se comprend très bien si l'on fait intervenir la notion de produit de solubilité. Nous n'insisterons pas davantage, dans cet article, sur la position de ce front. L'un de nous a commencé son étude détaillée.

\section{Déplacement du front.}

Dans le cas général, le front se déplace à mesure que la diffusion se poursuit, en laissant derrière lui une plage rectangulaire de précipité. Si le rapport $\mathrm{C}_{1} / \mathrm{C}_{2}$ est de $1 / 10$, on peut observer un déplacement de l'ordre de $5 \mathrm{~mm}$.

\section{Front de régime permanent.}

Au bout d'un temps assez élevé, le front se fixe définitivement et le régime permanent s'établit. Il est probable que ce régime n'est vraiment atteint 


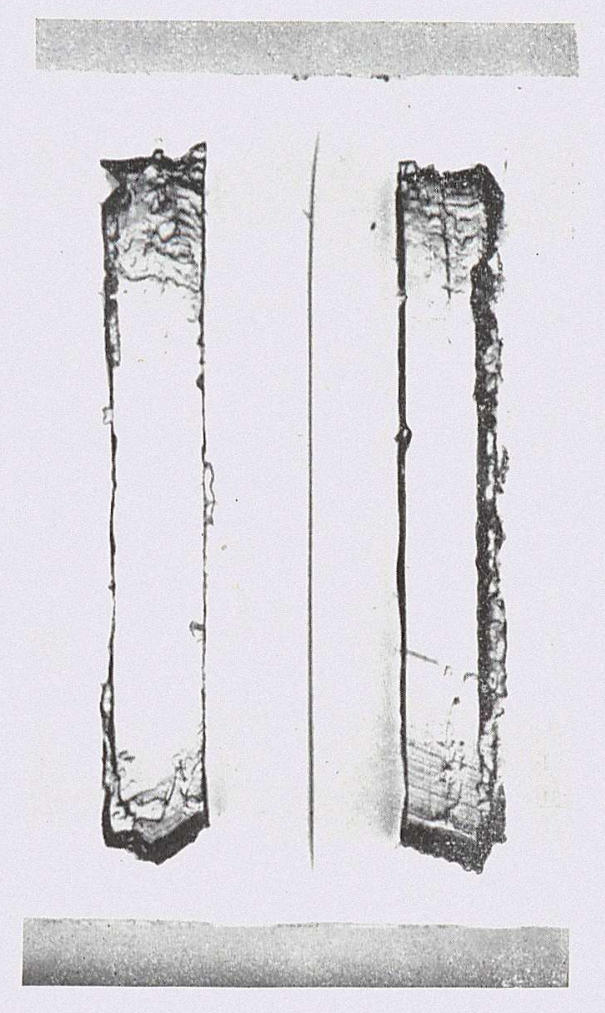

Photol.
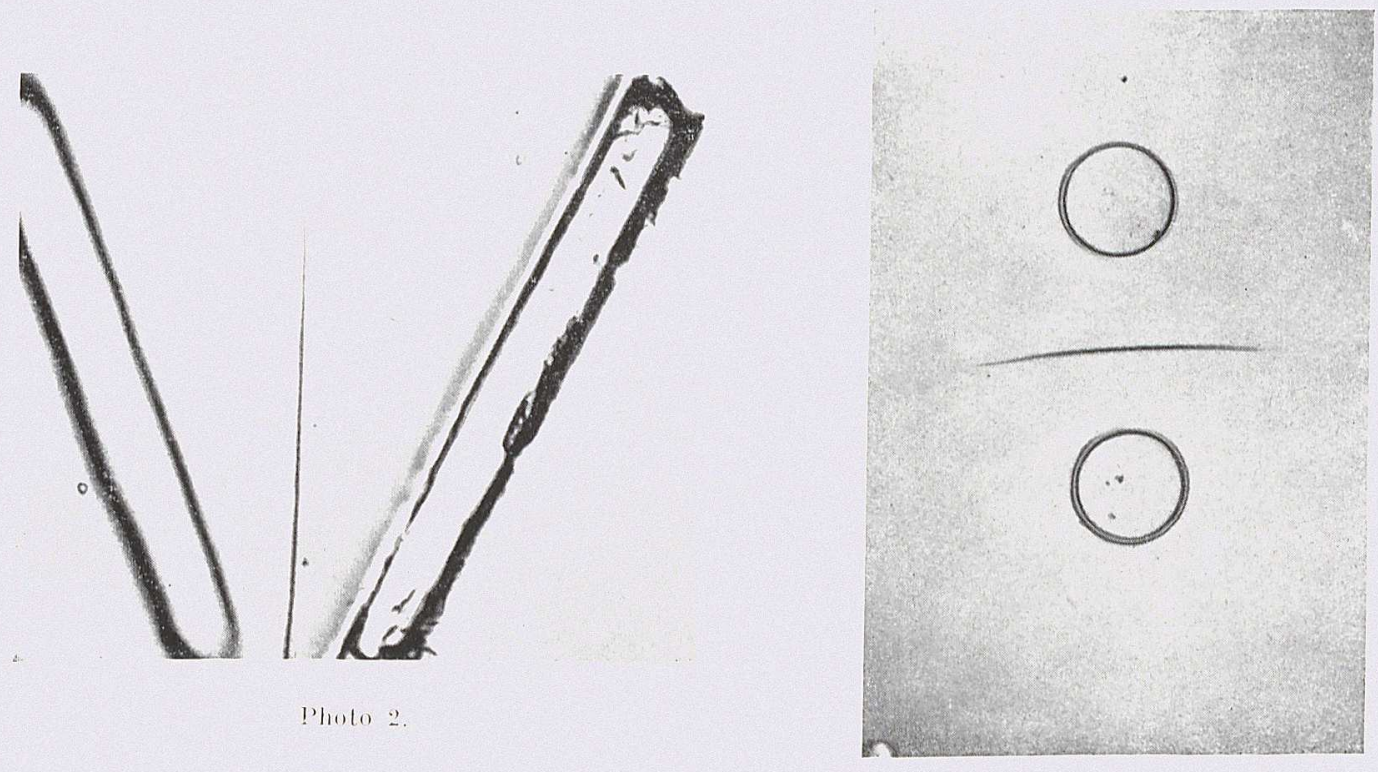

Photo 3. 
qu'au bout de trois ou quatre jours. Mais après un jour et demi ou deux jours, le front ne varie pratiquement plus. Il s'épaissit sur place.

Quand les concentrations en $\mathrm{SO}_{4} \mathrm{~K}_{2}$ et $\mathrm{Cl}_{2} \mathrm{Ba}$ sont égales, le front se fixe presque immédiatement. C'est le cas favorable à la mesure des coefficients de diffusion (photo 1). Dans les autres cas, l'un des réactifs doit traverser une plage de précipité, ce qui risque de modifier quelque peu son coefficient de diffusion.

\section{Résultats.}

La figure de diffusion de la photo 1 a été obtenue avec des solutions $1 / 4$ normales $\left(\mathrm{C}_{1}=\mathrm{C}_{2}=0,25 \mathrm{~N}\right)$. Le front rectiligne de précipité est nettement visible entre les deux réservoirs. Les bords en regard de ceux-ci paraissent bien plus nets au comparateur que sur la photographie où des effets de parallaxe sont inévitables. Les distances $\mathrm{QP}_{1}$ et $\mathrm{QP}_{2}$ (fig. 2) ont été mesurées au $1 / 10^{\mathrm{e}} \mathrm{mm}$. près. Le rapport $\mathrm{D}_{1} / \mathrm{D}_{2}$ est égal à 0,87 .

\section{$\left.2^{\circ}\right)$ Domaine angulaire.}

Nous avons procédé comme dans le cas précédent. Mais les réservoirs font entre eux l'angle $\alpha$ (voir photo 2 où le manque de netteté des réservoirs est dû à la présence des solutions). Leur découpage se fait en posant la plaque gélatineuse sur un papier millimétré où leur trace a été soigneusement dessinée. Après diffusion et formation du front permanent de précipité, la plaque est reportée sur le papier millimétré de telle sorte que les côtés $\mathrm{P}_{1} \mathrm{P}_{1}^{\prime}$ et $\mathrm{P}_{2} \mathrm{P}_{2}^{\prime}$ des réservoirs coïncident à nouveau avec leurs traces. Le front QQ' est repéré sur le papier. Les angles $\left(\mathrm{OP}_{1}, \mathrm{OQ}\right)$ et $\left(\mathrm{OQ}, \mathrm{OP}_{2}\right)$ sont mesurés au rapporteur. On a trouvé :

$$
\mathrm{D}_{1} / \mathrm{D}_{2}=0,84
$$

30) Domaine extérieur à deux cercles.

Nous avons fait une étude expérimentale plus poussée de ce cas intéressant.

Les réservoirs sont constitués par deux tubes cylindriques égaux de laiton, posés sur la lame gélatineuse. Leurs extrémités en contact avec le gel sont travaillées au tour, très minces et soigneusement rodées. Les deux tubes sont rigidement unis, de sorte que les bases géométriques de la figure de diffusion sont connues une fois pour toutes. Les réactifs $R_{1}$ et $R_{2}$ pénètrent très rapidement dans la lame et effectuent ensuite des diffusions cylindriques à partir des traces circulaires $S_{1}$ et $S_{2}$ des tubes sur la lame (photo 3).

Cependant, en s'enfonçant dans la lame, les réactifs contournent légèrement les bords des tubes. Il en résulte que les cercles $S_{1}$ et $S_{2}$ ne sont pas aussi parfaitement définis qu'on pourrait le croire. Mais, comme il s'agit de mesures relatives, l'erreur qui en résulte est certainement faible.

Faisant varier la concentration commune $\mathrm{C}_{1}=\mathrm{C}_{2}$ dans d'assez larges limites, nous avons trouvé des rapports $D_{1} / D_{2}$ compris entre 0,86 et 0,89 .

\section{Conclusion.}

Bien que les deux premiers montages expérimentaux soient un peu rudimentaires, les formules (29) (30) et. (36) sont convenablement vérifiées. Les résultats sont homogènes et en accord avec ceux que l'on obtient par d'autres méthodes.

En dehors de la mesure des coefficients de diffusion, notre étude peut avoir plusieurs applications qui seront précisées par la suite.

Faculté des Sciences de Montpellier. Institut de Chimie. 